\title{
Erratum: The structure of a polyQ-anti-polyQ complex reveals binding according to a linear lattice model
}

Pingwei Li, Kathryn E Huey-Tubman, Tiyu Gao, Xiaojun Li, Anthony P West, Jr, Melanie J Bennett \& Pamela J Bjorkman Nat. Struct. Mol. Biol. 14, 381-387 (2007); published online 22 April 2007; corrected after print 8 May 2007

In the version of this article initially published, the incorrect corresponding authors were listed. The corresponding author should be Pingwei Li (pingwei@neo.tamu.edu).We apologize for this mistake.

In addition, a paper was not cited. The missing citation is: 49. Altschuler, E.L., Hud, N.V., Mazrimas, J.A. \& Rupp, B. Random coil conformation for extended polyglutamine stretches in aqueous soluble monomeric peptides. J. Pept. Res. 50, 73-75 (1997).

The citation should appear on page 381, in the eighth sentence of the article's second paragraph, as follows: "However, studies involving polyQ peptides $^{14,26-28,49}$, and our studies using polyQ tracts in the context of the HD exon 1 protein ${ }^{29}$, demonstrated that the predominant species of both normal and expanded unaggregated polyQ in solution is an extended random coil and showed no evidence for a detectable population of expanded soluble polyQ molecules with global conformational differences from normal polyQ."

These errors have been corrected in the HTML and PDF versions of the article.

\section{Corrigendum: Shifting nanoscopic clock gears}

\section{Susan S Golden, Vincent M Cassone \& Andy LiWang}

Nat. Struct. Mol. Biol. 14, 362-363 (2007).

In the version of this News and Views initially published, the following sentence was missing from the legend to Figure 1: "Figure scheme based on a design by S.R. Mackey."

\section{Corrigendum: FtsK: A groovy helicase}

Terence R Strick \& Audrey Quessada-Vial
Nat. Struct. Mol. Biol. 13, 948-950 (2006).

In the version of this News and Views initially published, the authors present a model to explain the low degree of positive DNA supercoiling generated during DNA translocation through the FtsK helicase. The authors' conclusion, that in a rotary inchworm model for DNA translocation the helicase should generate positive supercoils ahead of itself when taking steps of less than 1.7 base pairs, is incorrect. Positive supercoils should be generated by steps of more than 1.7 base pairs, as described by Massey et al. (Mol. Cell 23, 457-469, 2006) and as pointed out to the authors by Dr. Ian Grainge. The authors deeply regret this error and apologize for any misunderstandings it may have caused. 